Stadtpolitik und kommunale Planung im Westen

\title{
Wechselnde Stimmungen
}

\section{Die Entwicklung des städtischen Verkehrs in Bremen zeigt, wie sehr die ver- kehrlichen Leitbilder in Abhängigkeit von Politik, Einzelinteressen und Inter- essen einem stetigen Wandel unterliegen. Die Bürger der Stadt zeigen sich jedoch weitgehend zufrieden.}

A Von Michael Glotz-Richter ls Hafenstadt ist die Geschichte und Gegenwart Bremens eng mit der Rolle als Umschlagplatz verknüpft. Die Veränderungen in der Standortbewertung haben die Stadt Bremen als ehemaligen Hafenstandort für mitteldeutsche Regionen in eine eher periphere Lage gebracht. Auch sechs Jahre nach der ,Wende“ ist die Wiederherstellung der direkten Schienenanbindung in Richtung Berlin (über Uelzen-Salzwedel) nur in Planung, und in der Schienenverbindung zwischen Hamburg und dem Ruhrgebiet kann man Bremen sprichwörtlich links liegen lassen und ohne Halt daran vorbeifahren. Gleichzeitig wird der Straßenverkehr in der Region ständig erweitert: vom sechsstreifigen Ausbau der A1 über den Neubau der A 28 (Oldenburg - Delmenhorst) bis hin zu diversen Ortsumfahrungen der Bundesstraßen im Umland.

\section{Dolitischer Wandel}

Ein im Vergleich zu anderen deutschen Großstädten zügiger Verkehrsfluß auf den Straßen lassen Bremen auf den ersten Blick als eine (noch) heile Verkehrswelt erscheinen, in der Stau eher herbeigeredet wird als denn vorhanden ist. Aktuell ist aber auch in Bremen - parallel zur politischen Entwicklung - eine Trendwende in der Verkehrspolitik unübersehbar.

Als sich nach jahrzehntelanger absoluter SPDMehrheit im Herbst 1991 eine Ampelkoalition etablierte, waren die Hoffnungen groß, eine Brücke zwischen Ökonomie und Ökologie zu schlagen. Doch sehr schnell haben sich die politischen Konflikte gerade auch im Bereich der Verkehrsplanung und Stadtentwicklung herauskristallisiert und zu politischen Blockadesituationen geführt. Im Frühjahr 1995 wurde der Ausstieg aus der Ampelkoalition eingeläutet.

Zeitgleich wurde die verkehrspolitische Diskussion in der Hansestadt zunehmend durch die „CityInitiative" geprägt. Mit mehreren ganzseitigen Anzeigen in der Sonntagsausgabe der bedeutendsten Bremer Tageszeitung machten die darin ver- tretenen Einzelhändler und Verbände mobil gegen eine in ihren Augen zuwenig auf das Auto ausgerichtete Verkehrsplanung. In der vorgezogenen Bürgerschaftswahl vom Mai 1995 verlor die SPD schließlich erheblich an Stimmen und regiert nun gemeinsam mit der CDU in einer großen Koalition.

\section{- Verkehrssituation}

Heute stellt sich die Verkehrssituation in Bremen widersprüchlich dar. Mit einem Anteil von 22 Prozent Fahrradverkehr, 18 Prozent ÖPNV, 21 Prozent Fußgängerverkehr werden rund 60 Prozent aller Wege der Bremer Bevölkerung mit dem Umweltverbund zurückgelegt. Aus dem Umland kommen rund 86 Prozent der Einpendler mit dem Kraftfahrzeug, was die Automobilabhängigkeit des dünn besiedelten Umlandes besonders deutlich zeigt.

Die Bremer Straßenbahn BSAG hat in den vergangenen Jahren ihren Wagenbestand modernisiert und 78 Niederflurbahnen in Betrieb genommen. Hiermit ist nun die Hälfte des Strassenbahnfuhrparks benutzerfreundlich niederflurig, im Busfuhrpark sind es nahezu 100 Prozent. Zusammen mit anderen Maßnahmen des Kundenservices, der Information und des Erscheinungsbildes konnte der Bremer ÖPNV seine Attraktivität erheblich verbessern. Die aktuelle Socialdata-Erhebung zeigt, daß 59 Prozent der BremerInnen mit dem ÖV-Angebot zufrieden sind, nur zwölf Prozent unzufrieden.

\section{Erreichbarkeit der Innenstadt}

Der im Bundesvergleich unterdurchschnittliche Zentralitätsfaktor der Kaufkraftbindung aus der Region (vom Wirtschaftsressort geschätztes „Umsatzdefizit“ eine Milliarde pro Jahr) hat die verkehrspolitische Diskussion in Bremen sehr stark auf die „Verbesserung der Erreichbarkeit des Oberzentrums Bremen“ als Teil des wirtschaftspolitischen Sanierungsprogramms focussiert. Hierbei wird davon ausgegangen, daß beispielsweise rund 70 Prozent des Einkaufsverkehrs mit dem PKW stattfinden soll, und dem- entsprechend auf die Folgewirkung von Verbesserungen für den MIV gehofft. Aus- und Neubau von Parkhäusern in der Innenstadt werden politisch ebenso als Teil des Programmes zur Haushaltssanierung bewertet wie $\mathrm{z} . \mathrm{B}$, der Bau eines Tunnels zur besseren Anbindung der Gewerbeareale in Sebaldsbrück an die Autobahn. Dieser mit zwei Röhren und vier Fahrspuren geplante Tunnel ist aus Bremischen Mitteln zu finanzieren. Die aktuell geschätzten Baukosten belaufen sich auf 300 Millionen Mark. Damit suvbentioniert die Stadt jedes der 17.000 prognostizierten durchfahrenden Autos mit fast fünf Mark subventioniert.

Für die Verbesserung in den bestehenden innersțädtischen Wohngebieten wurde und wird stark auf die Nutzung von Car-Sharing gesetzt. Rund sechs Jahre nach ihrer Gründung kann das organisierte Car-Sharing in Bremen mit etwa 1000 NutzerInnen und etwa 55 Fahrzeugen an 31 Standorten viele Optionen zur Entlastung der verdichteten Quartiere vorweisen. Projekte wie „Minus 100“ (Wegfall von 100 illegalen Stellplätzen) sollten parallel zu Pollersetzungen über Kombinationsangebote von Car-Sharing und ÖPNV eine Alternative zum eigenen Auto aufzeigen. Zwölf Autofahrer wechselten im Angebotszeitraum (Frühjahr 1996) vom eigenen Auto auf diese Alternative. Wie auch bei anderen Projekten zeigte sich die Abhängigkeit von der Wirksamkeit von Alternativangeboten zum Auto zu Restriktionen für das Auto.

Doch Restriktionen für den Autoverkehr sind immer weniger angesagt in einer Stadt, die auf die klassischen Werte der automobilen Erreichbarkeit zur Verbesserung ihrer Standortgunst setzen will. Und dieses, obwohl doch mit höheren Kosten für den Straßenverkehr Bremen seine Standortqualitäten als Umschlagplatz zwischen Wasser, Schiene, Straße und Luft erhöhen würde.

Die Distanz zwischen Forschung - wie sie auch mit dem BMBF-Projekt zu stadtökologisch verträglicher Mobilität stattfindet - und politischem Steuern im Feld der Ökologie scheint sich tendenziell eher zu vergrößern als zu verringern. Hier sind auch im Projekt „Stadtökologie“ des Bundesforschungsministers erhebliche Anstrengungen in Hinblick auf einen verbesserten Praxisbezug nötig.

\section{Der Autor}

Michael Glotz-Richter, Dipl.-Ing., tätig beim Senator für Fraven, Gesundheit, Jugend, Soziales und Umweltschutz, Hanseatenhof 5, 28195 Bremen,

Tel. (0421) 361-6703 
(c) 20I0 Authors; licensee IÖW and oekom verlag. This is an article distributed under the terms of the Creative Commons Attribution Non-Commercial No Derivates License (http://creativecommons.org/licenses/by-nc-nd/3.o/), which permits unrestricted use, distribution, and reproduction in any medium, provided the original work is properly cited. 